\title{
Stellaria chilensis Pedersen en Chile y su sinonimia
}

\section{Stellaria chilensis Pedersen in Chile and its synonymy}

\author{
Carola Regina Volponi
}

Museo de La Plata, Facultad de Ciencias Naturales y Museo, Universidad Nacional de La Plata.

Casilla de correo 312, Correo Central, B1900AVW - La Plata, Argentina.

carolarv@fcnym.unlp.edu.ar

\begin{abstract}
Three species of Stellaria living in Chile, S. serpens Ravenna, S. circinata Ravenna and S. concinna Ravenna are synonymized with $S$. chilensis. It is considered that the features taken into account by Ravenna to establish such species, mainly plant habit and morphology of styles, are within the range of variability of $S$. chilensis. It is considered that $S$. celsa do not belong to this genus.
\end{abstract}

En las especies de Stellaria que crecen en Argentina y Chile, las semillas presentan suficientes caracteres en la ornamentación de la cubierta seminal de la cara tangencial externa como para diferenciarlas (cfr. Volponi 1986a, 1986b, 1992, 1993, 2006b). Otros elementos utilizados para diferenciar estas especies son la morfología de sépalos y pétalos, la relación de tamaño sépalos/pétalos, forma del gineceo y dehiscencia de la cápsula (Volponi 1986a, 1992, 1993, 2006b). Las Stellaria son hierbas anuales, bianuales o perennes, frágiles, apoyantes, decumbentes.

El objetivo del presente trabajo es fundamentar la sinonimización de tres especies de Stellaria, S. serpens Ravenna, S. circinata Ravenna y S. concinna Ravenna, con $S$. chilensis Pedersen y se agregan observaciones acerca de S. celsa Ravenna.

Para este trabajo se estudiaron los siguientes ejemplares: Stellaria chilensis Pedersen: CHILE. Prov. Aconcagua, Petorca, Pichicuy, ladera oeste de los cerros, frente a la playa, 5-20 msm, 1-XI-1974, 32 $20^{\prime} \mathrm{S}$; $71^{\circ} 28^{\prime} \mathrm{W}$, Marticorena et al. 196, CONC 43004 (Fig. 1 D); Prov. Concepción, camino entre Concepción y Coronel, km 15,11-X-1967, Villarroel \& Weldt 98, CONC 35088 (Fig 1 C);

Stellaria serpens Ravenna: CHILE. Región Metropolitana; Cordillera; in decliviis vallis fluminis Maipo ad viam Torrecillas del Manzano. Ravenna, P. F. 5437 SI 089804!, Isotipo SI004323 (Fig. 1 A-B).

De las especies $S$. circinata, $S$. concinna y $S$. celsa sólo se han consultado las descripciones originales.

El método empleado para la observación de las semillas, tanto del isotipo de $S$. serpens como de $S$. chilensis es el mismo. Se extrajo una sola semilla de la cápsula, fue observada con un microscopio estereoscópico Leica MS5, luego fue montada con adhesivo sobre un círculo metálico para ser metalizada con oro-paladio y observada y fotografiada: la de $S$. serpens con un microscopio electrónico de barrido JEOL JSM 6360LV SEM (MEB) (Fig. 1 A-B); mientras que la de $S$. chilensis fue asimismo observada y fotografiada con un microscopio electrónico de barrido JEOL JSM U3 (cfr. Volponi 1986b, pág. 284) (Fig. 1 C-D).

Los acrónimos BA, CONC, CTES, SI, P están de acuerdo con Holmgren et al. (1990).

En la Tabla I se comparan las descripciones de Stellaria chilensis de Pedersen (1983), descripción original; la de Volponi (1993), y la de Stellaria serpens Ravenna (2006b).

A) La SinOnimización de STELLARIA SERPENS RaVEnNa

En una visita al herbario SI se encontró un isotipo de Stellaria serpens Ravenna (SI004323), que está en condiciones adecuadas para ser identificado correctamente. De él se extrajo una semilla de una cápsula ya dehiscente. Al observar dicha semilla bajo microscopio estereoscópico se constató que la cubierta seminal estaba ornamentada por gloquidios (Fig. $1 \mathrm{~A}, \mathrm{~B}$ ). Teniendo en cuenta lo dicho más arriba, resultó llamativo, ya que la única especie de Stellaria que presenta este tipo de ornamentación es $S$. chilensis Pedersen (Fig. 1 C, D) (cfr. Volponi 1993).

Por otra parte, Volponi (1993) describe y menciona a Stellaria chilensis entre Perú, Santiago de Chuco, ca. $8^{\circ}$ de latitud Sur y Chile, Isla Quiriquina, ca. $37^{\circ} \mathrm{S}$.

En el isotipo en SI dice, de puño y letra de Ravenna: "Collections. In umbrosis decliviis vallis fluminis Maipo, ad vim Torrecillas del Manzano, Andibus Chiliae; Ravenna 5437, Nov. 2006 (BA typus! Herb. Rav. et caet. isotypi)" (Ravenna, 2006b: 2). Por otra parte, en http://plants.jstor. org/specimen/si004323, en "Notes", se lee lo siguiente: "La etiqueta no indica número del herbario Ravenna. Sin embargo, la localidad de colección se corresponde con la indicada en el protólogo y de puño y letra de Ravenna se indica "Isotypus". Protólogo publicado en Onira 11(1): 
1. 2006." El cerro Torrecillas, en Chile, se encuentra en $33^{\circ} 34^{\prime} 11^{\prime \prime} \mathrm{S} ; 70^{\circ} 23^{\prime} 01^{\prime \prime} \mathrm{W}$.

Se debe mencionar que no se ha encontrado el "typus" de Stellaria serpens en el herbario del Museo Argentino de Ciencias Naturales "Bernardino Rivadavia" (BA), citado por Ravenna (1.c.).

Pedersen (1983) y Volponi (1993) describen la cápsula y la semilla de $S$. chilensis (Tabla I), mientras que Ravenna (2006b: 1) omite la descripción de estos caracteres en $S$. serpens y el isotipo presenta una cápsula con semillas. Asimismo, este isotipo no está citado en el protólogo de Stellaria serpens, cuya etiqueta de herbario está manuscrita por Ravenna; que no se ha encontrado ninguno de los isotipos y/o paratipos citados por Ravenna en los herbarios mencionados. Por lo que antecede se concluye:

Stellaria chilensis Pedersen, Bonplandia 5 (22): 204, 1983. Sinónimo: Stellaria serpens Ravenna, Onira 11 (1): 1, 2006.
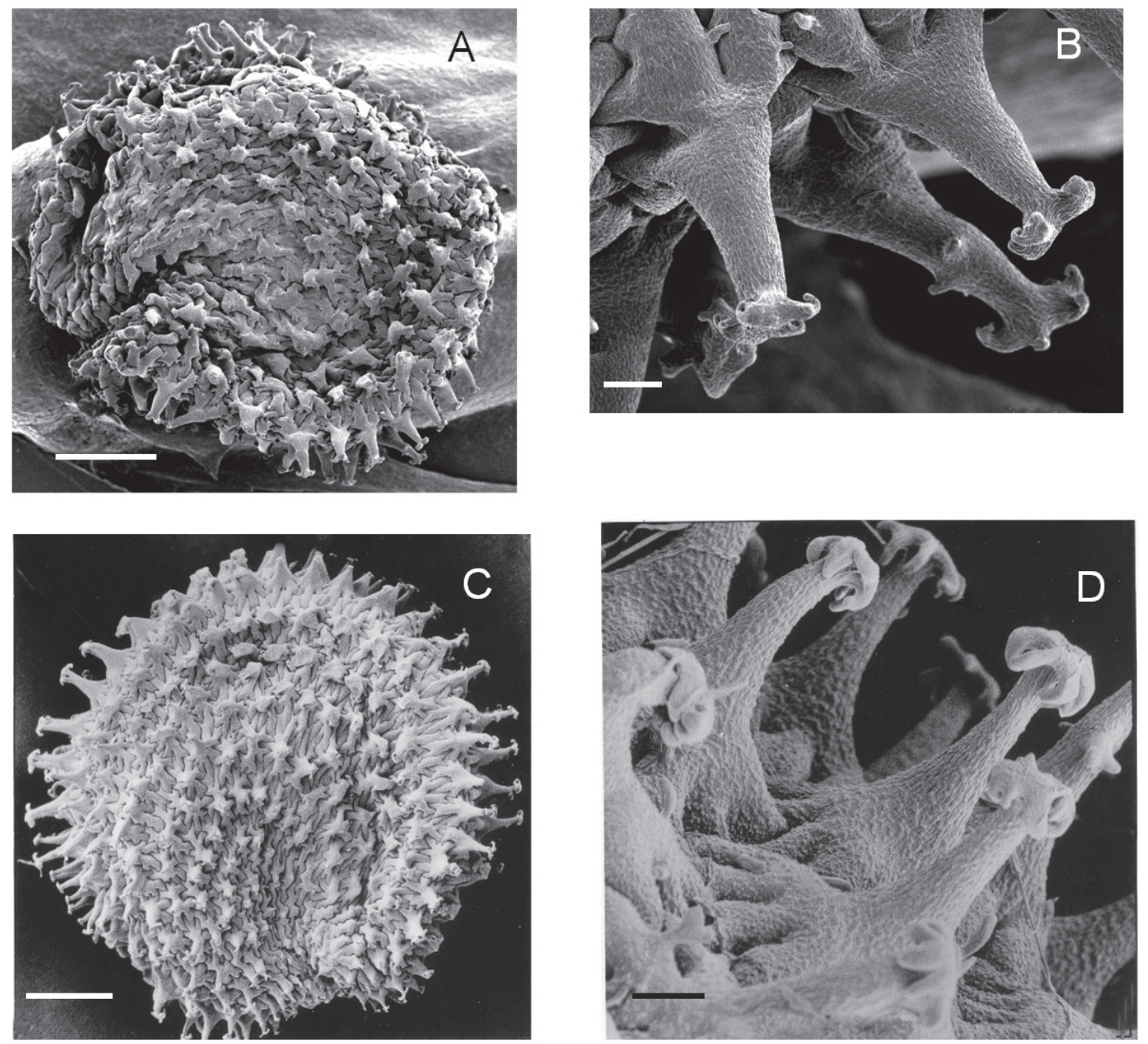

Figura 1. A-D MEB. A, vista lateral de una semilla extraída del isotipo de Stellaria serpens Ravenna (Ravenna 5437 SI) ; B, detalle de la misma semilla mostrando las evaginaciones de las células de la cubierta seminal terminadas en gloquidios; C, vista lateral de una semilla de Stellaria chilensis Pedersen (CONC 35088); D, detalle de una semilla de S. chilensis mostrando las evaginaciones de las células de la cubierta seminal terminadas en gloquidios (CONC 43004). Escalas: A, C = $200 \mu \mathrm{m}$; B, D = $20 \mu \mathrm{m}$.

Figure 1. A-D SEM. A, side view of a seed taken from the isotype of Stellaria serpens Ravenna (Ravenna 5437 SI); B, detail of the same seed showing evaginations of the seedcoat terminated in glochidia; C, side view of a seed of Stellaria chilensis CONC 35088 Pedersen; D, detail of a seed $S$. chilensis showing seedcoat cells terminated in glochidia (CONC 43 004). Scales: A, C = $200 \mu \mathrm{m}, \mathrm{B}, \mathrm{D}=20 \mu \mathrm{m}$. 
Stellaria chilensis y su sinonimia: VolPONI, C.

Tabla I. Comparación de las descripciones, la original de Stellaria chilensis Pedersen, y de Volponi (1993) con la descripción original de S. serpens Ravenna.

TABLE I. Comparison of descriptions, the original Stellaria chilensis Pedersen, and Volponi (1993) with the original description of S. serpens Ravenna.

\begin{tabular}{|c|c|c|c|}
\hline & $\begin{array}{l}\text { Stellaria chilensis Pedersen, } \\
\text { descripción original }\end{array}$ & $\begin{array}{l}\text { Stellaria chilensis Pedersen, } \\
\text { Volponi } 1993 .\end{array}$ & $\begin{array}{l}\text { Stellaria serpens Ravenna, descripción } \\
\text { original }\end{array}$ \\
\hline Hábito & $\begin{array}{l}\text { hierba perenne?, decumbente } \\
\text { (raíz no vista) }\end{array}$ & herbácea perenne? hemicriptófita? & Reptante, herbácea \\
\hline Tallo & $\varnothing 0,2-0,25 \mathrm{~cm}$ & $\varnothing 1-3,5 \mathrm{~mm}$ & frágil, glabrescente, $20-100 \mathrm{~cm}$ largo \\
\hline Entrenudos & -------- & 20-170 mm largo & ---------- \\
\hline Pubescencia & mayor densidad hacia el ápice & $\begin{array}{l}\text { pelos de } 1 \mathrm{~mm} \text { largo, simples y } \\
\text { capitados }\end{array}$ & \\
\hline Hojas & $\begin{array}{l} \pm 4,5 \times 1,5 \mathrm{~cm} \\
\text { inferiores: pecioladas } \\
\text { superiores: sésiles }\end{array}$ & $\begin{array}{l}(17-) 20-45(-80) \times(9-) 11-33 \mathrm{~mm} ; \\
\text { inferiores: largamente pecioladas; } \\
\text { superiores: sésiles }\end{array}$ & $\begin{array}{l}\text { 18-30 x 13-19 mm; superiores: sésiles; } \\
\text { el resto, pecioladas. }\end{array}$ \\
\hline Hojas: forma & ovado-lanceoladas, acuminadas & $\begin{array}{l}\text { triangulares a oval triangular o oval } \\
\text { lanceolado; base a veces atenuada; } \\
\text { margen ciliado, ápice glabro. }\end{array}$ & $\begin{array}{l}\text { ovado lanceolada, herbácea, fláccida, } \\
\text { aguda }\end{array}$ \\
\hline Hojas: color & 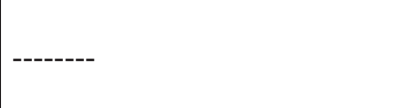 & $\begin{array}{l}\text { En general concoloras, raro } \\
\text { discoloras }\end{array}$ & verdes \\
\hline Hojas: pecíolo & |--------- & 4-20(-27) mm largo, pubescente & 2,5-16 mm largo, pubérulo \\
\hline Hojas: pubescencia & $\begin{array}{l}\text { ciliadas, pelos simples, con } \\
\text { pelos capitados mezclados, raro } \\
\text { glabra }\end{array}$ & --------- & |----------- \\
\hline Flores & $\begin{array}{l}\text { cima terminal, 5-6 veces } \\
\text { furcada }\end{array}$ & cima laxa & 7-14 flora, bifurcada, raro trifurcada \\
\hline Flores: pedúnculo & $\begin{array}{l}90 \mathrm{~mm} \text { largo, pubescencia } \\
\text { glandulosa; entrenudo 10-65 } \\
\text { mm largo }\end{array}$ & entrenudos 3-90 $\mathrm{mm}$ largo & $\begin{array}{l}\text { densamente pubescente, } 15-83 \mathrm{~mm} \\
\text { largo }\end{array}$ \\
\hline Flores: pedicelo & $\begin{array}{l}\text { 20-35 mm largo, glanduloso } \\
\text { pubescente, algunas veces } \\
\text { deflexos }\end{array}$ & $\begin{array}{l}\text { 12-48 mm largo; algunas veces } \\
\text { deflexos }\end{array}$ & gráciles, pubérulos, 10-13 mm largo \\
\hline Sépalos & $\begin{array}{l}\text { herbáceos, parte cubierta } \\
\text { escariosa; } 5 \text { a } 7 \mathrm{~mm} \text { largo }\end{array}$ & $\begin{array}{l}(5-) 6-10(-13) \text { x } 1-3 \mathrm{~mm} \text {, quizás } \\
\text { acrescentes en fruto }\end{array}$ & pilosos, 4,8-5,8 mm largo \\
\hline Sépalos: forma & $\begin{array}{l}\text { oblongo ovados, trinervados; } \\
\text { pubescencia glandulosa } \pm \\
\text { densa }\end{array}$ & herbáceos, ovado lanceolados & lanceolados, agudos \\
\hline Pétalos & $\begin{array}{l}10 \mathrm{~mm} \text { largo, bipartidos hasta } \pm \\
2 \mathrm{~mm} \text { de la base }\end{array}$ & $(9-) 11-14(-15) \mathrm{mm}$ largo & $\begin{array}{l}\text { corola infundibuliforme, blanca, 10-13 } \\
\text { mm largo }\end{array}$ \\
\hline
\end{tabular}




\begin{tabular}{|c|c|c|c|}
\hline Pétalos: lóbulos & $\begin{array}{l}\text { angostamente cuneados } \\
\text { espatulados con ápice } \\
\text { redondeado }\end{array}$ & $\begin{array}{l}\text { espatulados, márgenes divergentes, } \\
\text { ápice redondeado algo aguzado }\end{array}$ & $\begin{array}{l}\text { angostamente oblanceolados, 1,3-1,5 } \\
\text { mm ancho }\end{array}$ \\
\hline Estambres & 10; de 5-6 mm de largo & 10, tan largos como los sépalos & 5-3,8 mm largo \\
\hline Estambres: anteras & ovales de ca. $1 \mathrm{~mm}$ largo & $\begin{array}{l}\text { ovaladas, } 1 \text { mm largo; glándula } \\
\text { nectarífera visible }\end{array}$ & $\begin{array}{l}\text { versatiles, oblongo elípticas, } \\
\text { blancuzcas, 0,7-0,8 } \mathrm{mm} \text {, polen amarillo }\end{array}$ \\
\hline Estilos & 3 ; de 2,5 mm largo & $3 ; 2-3 \mathrm{~mm}$ largo & $\begin{array}{l}\text { erecto a veces ápice recurvado; } 1,8-2,3 \\
\text { mm largo }\end{array}$ \\
\hline Ovario & $\begin{array}{l} \pm 9 \text { mm largo, ca. } 40 \text { óvulos, } \\
\text { funículo breve, placenta } \\
\text { baculiforme }\end{array}$ & $2 \mathrm{~mm}$ largo & $\begin{array}{l}2,5 \times 1,5 \mathrm{~mm} \text {; ovado, verdoso; estigma } \\
\text { apical }\end{array}$ \\
\hline $\begin{array}{l}\text { Cápsula: } \\
\text { dehiscencia }\end{array}$ & $\begin{array}{l}6 \text { valvas en la parte superior; } 3 \\
\text { valvas en la parte inferior }\end{array}$ & $\begin{array}{l}\text { 10-14 mm largo, } 1,5 \text { veces más } \\
\text { larga que los sépalos; } 6 \text { valvas; } \\
\text { valvas curvadas hacia fuera en la } \\
\text { dehiscencia }\end{array}$ & --------- \\
\hline Semillas & $\begin{array}{l} \pm 1,2 \times 1 \times 0,6 \mathrm{~mm}, \text { de color } \\
\text { fuliginoso }\end{array}$ & $\begin{array}{l}1,2 \times 1 \times 0,5 \mathrm{~mm} \text {; casi orbiculares a } \\
\text { reniformes; de color castaño oscuro } \\
\text { a negras }\end{array}$ & --------- \\
\hline $\begin{array}{l}\text { Semillas: } \\
\text { ornamentación }\end{array}$ & $\begin{array}{l}\text { gloquidios de } 0,1-0,2 \mathrm{~mm} \text { de } \\
\text { alto }\end{array}$ & $\begin{array}{l}\text { largos gloquidios en la cara lateral y } \\
\text { el dorso; más cortos o ausentes en el } \\
\text { hilium }\end{array}$ & --------- \\
\hline TYPUS & Helsinki, H1636488, holotipo & -------------- & San Isidro, SI004323, isotipo \\
\hline
\end{tabular}

B) La sinonimización de Stellaria Concinna Ravenna y STELLARIA CIRCINATA RAVENNA

Se han comparado otras especies de Stellaria descritas por el mismo autor, $S$. concinna y $S$. circinata. De éstas, sólo ha sido posible analizar las descripciones originales, dado que los isotipos mencionados no están depositados en los herbarios BA, CONC, CTES, P. Por otra parte, ninguna de las especies propuestas ha sido ilustrada.

Los caracteres, hábito de la planta y morfología de los estilos, en que se basan las nuevas especies citadas más arriba, son extremadamente variables. Esta variabilidad se presenta en otras especies de Stellaria y en algunas de Drymaria (cfr. Volponi 2006a: 294). Así, el hábito de la planta depende de la cercanía de otras plantas y que se pueda apoyar, buscando la luz; en ese caso presentará entrenudos largos: "Plantae rupicolae delicatae" en Stellaria circinata (Ravenna 2005b). Si se desarrolla en praderas pequeñas muy luminosas, presentará un hábito más cespitoso, entrenudos más cortos: "Plantae reptantes, herbaceae" en S. serpens (Ravenna 2006b).

Por otro lado, la morfología de los estilos depende del grado de madurez de los mismos. Los estilos son filiformes, rectos y la superficie estigmática papilosa ocupa el ápice y parte del lado interno del estilo antes de la antesis; el ápice, a veces, está un poco curvado hacia afuera: "Styli erecti, filiformes, recti, ..., stigmatibus apicalibus minutis" en S. concinna (Ravenna 2005a). Una vez que han recibido los granos de polen, los estilos se alargan, se enroscan hacia afuera, porque ya no son más receptivos, son estilos senescentes: "Styli ... erecti, albi ... ad apicem extrorse circinati" en S. circinata (Ravenna 2005b).

Las localidades donde fue recolectado el material: S. circinata: Montenegro, prov. Chacabuco, Chile, 3258'0,74"S; 7050'17,88" W y S. concinna: Reserva Nacional Río Clarillo (Reserva natural estricta), Región Metropolitana de Santiago, Chile, 33⒋ ' S; 70²4' W, están dentro de los límites geográficos de la distribución de $S$. chilensis.

Después de considerar: a) las descripciones de las especies, comparándolas con la original de Stellaria chilensis Pedersen; b) que no se han encontrado ninguno de los isotipos y/o paratipos citados por Ravenna en los herbarios mencionados o bien son inexistentes; c) que la distribución geográfica está dentro de los límites de distribución de $S$. chilensis propuestos por Volponi (1993), se establece la siguiente sinonimia: 
Stellaria chilensis Pedersen, Bonplandia 5(22): 204, 1983 Sinónimos: Stellaria concinna Ravenna, Onira 9(14): 56, 2005; Stellaria circinata Ravenna, Onira 10 (11): 35-36, 2005.

C) Stellaria CElSA Ravenna, unA Determinación ERRÓnEA En Stellaria celsa, Ravenna (2006a) escribe para el hábito "Planta erecta usque $60 \mathrm{~cm}$ alta." y describe a los estilos como: "Styli erecti, tenuissiome filiformes, ..., ad apicem $0.3 \mathrm{~mm}$ abrupte patentes, stigmatibus minute capitatis". Volponi no ha observado ninguna especie de Stellaria en Chile (cfr. Volponi, Flora de Chile) ni en Argentina (Volponi, 1983, 1986a, 1986b, 1992, 1993, 2006b), cuyo porte sea erecto y que las papilas estigmáticas sólo se hallen presentes en el ápice de los estilos. Al igual que las especies mencionadas más arriba, el "typus" de $S$. celsa no se ha encontrado depositado en el BA.

Así, después de considerar: a) el hábito y la disposición de las papilas estigmáticas descriptas para Stellaria celsa por Ravenna (2006a); b) que al igual que en las especies citadas más arriba, no está ilustrada; c) que el "typus" no se encuentra depositado en BA; d) que Ravenna (2006a: 57) menciona un solo ejemplar, el "typus"; se concluye que es dudoso que el espécimen Ravenna 5340 (Ravenna 1.c.) pertenezca realmente a una Stellaria. Una inspección del material citado resolvería este problema.

\section{AGRADECIMIENTOS}

A Patricia Sarmiento, por su paciencia, amistad, dedicación y destreza en el manejo del microscopio electrónico de barrido del Museo de La Plata; a Norma Deginani y Manuel Belgrano, del SI, por la comprensión, seriedad y compromiso en el manejo del material de herbario; a Mirta Arriaga del BA; a Alicia Marticorena del CONC; a María Mercedes Arbo de CTES.

\section{BIBLIOGRAFÍA}

Holmgren, P. K., N. H. Holmgren \& L.C. Barnett, (eds). 1990. Index Herbariorum. $8^{\text {th }}$ edition, New York Botanical Garden, New York, X, 693 pp.

Pedersen, T. M. 1983. Two new species of Stellaria from South America, with a description of Stellaria arvalis F. Phil. Bonplandia 5(22): 203-210.

Ravenna, P. 2005a. Stellaria concinna sp. nov. and S. chilensis. Onira 9(14): 56-58.

Ravenna, P. 2005b. Stellaria circinata an additional new species from Central Chile (Caryophyllaceae). Onira 10(11): 3536.

Ravenna, P. 2006a. Stellaria celsa, a new species from the Andes of Central Chile (Caryophyllaceae). Onira 10(17): 5657.

Ravenna, P. 2006b. Stellaria serpens an additional new species from the Andes of central Chile (Caryophyllaceae). Onira 11(1): 1-2. 2006.

Volponi, C. R. 1983. Sinopsis de las especies argentinas de Stellaria (Caryophyllaceae). Lilloa 36: 69-75.

Volponi, C. R. 1986a. Sobre Stellaria cuspidata (Caryophyllaceae) y especies afines en Argentina. Kurtziana 18: 93-107.

VolPONI, C. R. 1986b. Contribución a la espermatología de especies argentinas de Stellaria (Caryophyllaceae). Boletin de la Sociedad Argentina de Botánica. 24: 283-294.

Volponi, C. R. 1992. Stellaria yungasensis (Caryophyllaceae), una nueva cita para Argentina. Anales de la Sociedad Cientifica Argentina. 222: 69-72.

Volponi, C. R. 1993. Stellaria cuspidata (Caryophyllaceae) and some related species on the Andes. Willdenowia 23: 193209.

Volponi, C. R. 2006a. Type and new combination for Stellaria lanceolata and a new synonymy for $S$. ratundifolia (Caryophyllaceae). Adansonia, sér. 3., 28(2): 287-298.

Volponi, C. R. 2006b. Stellaria aphanantha (Caryophyllaceae) en la provincia de Tucumán, Argentina. Lilloa 43: 87-91.

Recibido: 05.06 .12

Aceptado: 11.12 .12 